\title{
Matrix Structure: A Paradigm Choice for the Work of University Offices ZhangZhengjin ${ }^{1, a,{ }^{*}}$
}

${ }^{1}$ Law collage OF Minjiang University, minhou, fuzhou, fujian, china a32659614@qq.com

${ }^{*}$ Corresponding author

Keywords: Matrix Structure, University Office, Work Operation, Paradigm Choice

\begin{abstract}
In view of the contradictions existing in university offices, this paper comprehensively applies the theory of higher education management and psychology of school management, and proposes the matrix structure as a paradigm choice for the work of university offices from the perspective of organizational structure.Meanwhile this paper provides materialist dialectics,the qualitative operation of time,contingency theory and performance appraisal as the tools for building a matrix-structured university office.
\end{abstract}

\section{矩阵结构：高校办公室工作运行的一种范式选择 \\ 张正金 $1, \mathrm{a},{ }^{*}$ \\ 1 闽江学院法学院, 闽侯, 福州, 福建, 中国 a32659614@qq.com \\ *通讯作者}

关键词：矩阵结构; 高校办公室; 工作运行; 范式选择

中文摘要.本文从组织结构的角度切入, 综合运用高等教育管理学、学校管理心理学的理论, 针对高校办公室的矛盾存在, 提出矩阵结构是高校办公室工作运行的一种范式选择, 并提供 唯物辩证法、时间的定性运筹术、权变理论、绩效考核作为构建矩阵结构型高校办公室的工 具选择。

\section{1.引言}

高校办公室是高校的综合办事机构，在全校的管理体系中处于中枢位置，其决策的优劣、 工作效率的高低, 将直接影响到全校工作的运转。组织结构作为影响办公室工作效能的重要 因素, 其设计是否合理, 对办公室的工作运行起着至关重要的作用。

\section{2.矩阵结构的涵义及其应用}

\section{1矩阵结构概述}

“矩阵”是一个数学概念, 最初由解线性方程组产生。数学上把多个互相关联的元素按一 定规律排成横行、纵列的一个矩形, 称为“矩阵”。将矩阵概念引用到组织范畴, 便产生了矩 阵结构的组织形式。1

\footnotetext{
1 叶聚丰,沈勤.矩阵式结构理论及其在高校体制建设中的应用 $[J]$. 大学教育研究, 2008: (2). 7-8
} 
矩阵结构以项目和任务为导向, 是一种同时进行若干个项目和任务管理的组织结构形式。 矩阵结构在科学研究、军事、工业等领域有着广泛应用, 尤其是在航空航天领域发挥着重要 的作用。矩阵型组织综合了职能型组织和项目型组织的优点, 在发挥职能部门纵向优势的同 时也发挥项目组织横向优势, 不但满足了对资源、技术的要求, 而且实现了对项目任务快速 应对的需要。

矩阵结构中的职能部门是固定的, 而项目小组是临时的; 职能部门负责为项目的成功提 供所需的资源和支持, 项目小组则负责项目的攻克。因此, 非常适用于综合活动团队作战和 临时攻关项目。另一方面, 由于参加项目的人员都来自不同的部门, 其归属关系仍在原部门, 这导致了项目负责人对项目组成人员管理上存在着困难。这种人员上的双重管理是矩阵结构 的先天不足和缺陷, 需要靠组织负责人的协调沟通以及积极向上的部门文化来弥补。

\section{2高校办公室工作和科室设置的特点}

高校办公室作为学校综合协调部门, 发挥着综合、协调、参谋、服务、督促等职能, 起 着上传下达的重要作用。一般都要承担综合文字、信息报送、督查、机要、会务接待、信访、 信息公开、应急值班等工作。高校办公室的工作特点是“重、苦、杂、繁”, 综合性强, 涉及 面广, 临时性和突发性事务多, 经常加班加点, 既要主动服务, 更要被动服务, 有时甚至吃 力不讨好，招人误解和非议。

高校办公室机构设置较政府办公室要显得简单和扁平。政府办公室一般同时设有政务机 构与专业机构, 政务机构履行办公室日常职责; 而专业机构为某一领域专设, 其职能较为单 一, 主要是作为分管领导的办公机构同时也履行政务机构的部分职能。一般来说, 政府办公 室内设督查、综合、秘书、文电等等政务科室和文教、商管、农业、政法、城建等专业科室, 除此外, 还有不少挂靠的单位, 其职能呈现网状分布, 应有尽有。而高校办公室内设机构则 较为直观简单, 通常只设综合、行政、督查、机要等科室, 人员相对较少。与政府办公室相 比之下, 高校办公室所承担的工作与任务就显得更为繁和杂, 对于人员的全面素质要求更高, 更需要几个一专多能、能单打独斗的人才。

\section{3矩阵型组织结构在高校办公室工作中的运用}

随着高等教育的不断纵深发展, 高校办公室的工作内容和工作结构也发生了巨大的变化, 这种在高校办公室主任直接领导下设置科室, 同时再根据实际情况成立临时项目小组的组织 结构越来越显示出其优势并成为发展趋势（如图1所示）。 


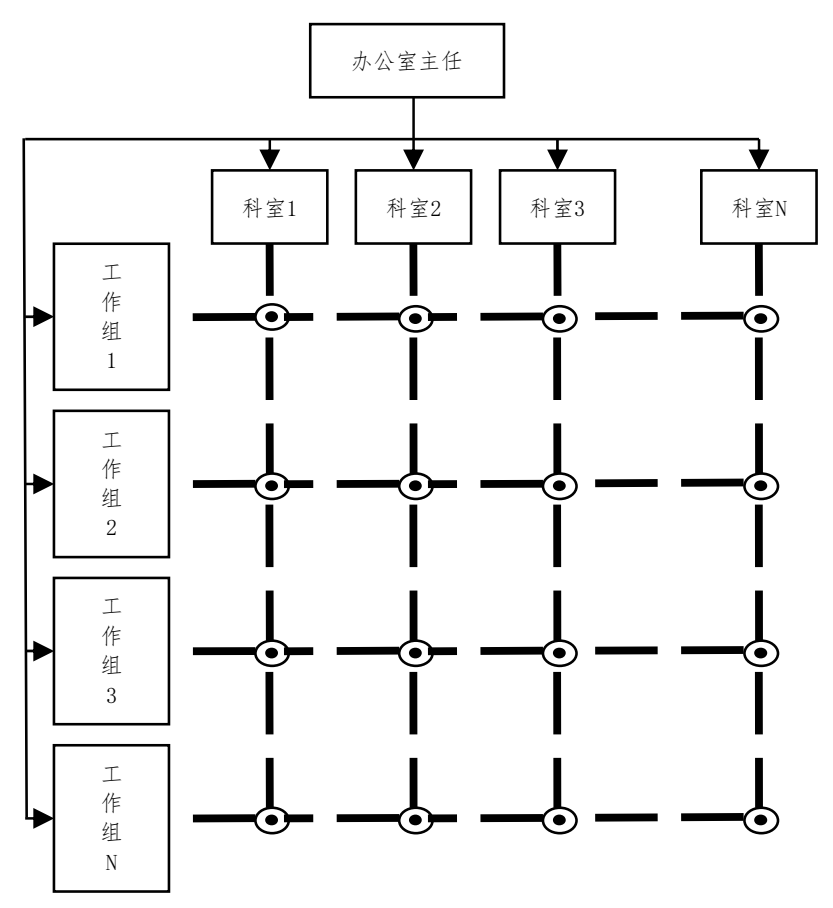

图1 矩阵型高校办公室

项目小组的人员是根据项目和任务的需要由办公室负责人在办公室内各科室中遴选组 成, 这些人员既隶属项目小组负责人领导, 又接受其来源科室的领导, 在纠正各科室横向联 系差、缺乏弹性的同时可以最大限度地利用有限的人力资源, 集中几个科室的优势, 在短时 间内快速有效处理突发任务和项目。比如, 根据工作实际需要, 临时组建校运动会项目小组、 开学典礼项目小组、毕业典礼项目小组、迎接校外来宾来校调研项目小组等, 从办公室内不 同科室抽调人员组成项目小组, 成员分别负责文稿、会务、接待、协调等工作, 由项目负责 人负责总协调, 这个项目负责人可以视工作内容而临时指定, 也可以相对固定, 毕竟高校有 其内生规律, 不少工作每年具有重复性。需要注意的是, 项目小组负责人、成员可以在原科 室和不同的项目小组之间同时工作。

\section{3. 高校办公室推行矩阵结构的必要性}

\section{1高校办公室工作的矛盾存在}

3.1.1整体与局部的矛盾存在

整体与局部是辩证统一的关系，二者既相互联系又相互区别。整体居于主导地位，局部 服从和服务于整体。整体是由局部构成的, 整体离不开局部; 局部是整体中的局部, 只有存 在于整体中, 局部才具有他的功能。高校办公室是由各科室和各项任务等要素构成的统一整 体, 其功能是以其整体功能而论, 而不是各个科室功能的简单相加。各个科室只有在办公室 整体功能的指引下, 为了完成具体项目和任务, 以合理的方式相互联系在一起并发挥各自的 功能, 才能保证办公室整体功能的实现。

3.1.2分工与协作的矛盾存在

分工是技术进步和生产社会化的产物, 通过明确具体的工作范围和职责, 可有效提高工 作效率和专业化程度。而协作的过程是“交换”的过程, 是内部的协调与配合。由于分工, 形 成科室、工作人员之间相对独立的局面, 有时候甚至出现摩擦、冲突, 分工似乎与协作是不 可共存的。但是办公室整体工作的提高, 如果光是依靠完成分工负责的任务肯定是难以完成 
的。分工和协作都是办公室整体工作中不可缺少的有机组成部分, 分工是协作的基础, 协作 是分工的上升。平时常说的“分工不分家”“不拆台、多补台”说的就是这个道理。

3.1.3广度与深度的矛盾存在

广度与深度之间是对立统一的关系。只有广度而没有深度，工作只停留在表面；只有深 度而没有广度, 工作也难以全面铺开。一定的深度要以一定的广度为基础, 越是要求高尖, 就越需要广博。对工作的要求如此, 对办公室队伍建设的要求亦是如此。一般来说, 人们在 求学时更偏知识的广度, 刚工作阶段则是在某个专业领域深度上钻研, 到了一定程度又从深 度钻研上转为广度的拓展和交叉融合。

\section{2矩阵结构型高校办公室的角色扮演}

3.2.1矩阵结构型高校办公室是整体和局部的“设计师”

矩阵结构型高校办公室通过横向、纵向排列组成矩阵结构, 实现工作网格化, 能够提供 给办公室负责人一个整体的视野。通过立足全局, 从整体着眼, 根据项目和任务的性质, 可 以拟定最优工作方案, 精挑细选人员组成工作组。成员既同原科室保持组织与业务上的联系, 又参加项目小组的工作, 既照顾了局部, 又服从整体。在临时项目小组里, 由于目的明确, 能够把自己的项目工作同整体工作联系在一起, 从而实现攻艰克难、完成任务。项目和任务 一旦完成, 又可以快速解散, 重新组合形成新的工作组, 以局部项目的高效完成和快速组合 不断推进整体工作。

3.2.2矩阵结构型高校办公室是分工和协作的“调度员”

科学的调度过程是一个集权和分权最优结合的过程。科学有效的调度可以加强不同科室 之间的互通和配合, 能够避免直线职能结构中各科室相对独立、各成体系的问题。相对职能 式结构来说, 矩阵结构下常规工作和临时工作一目了然, 可以减少工作层次与决策环节, 通 过二次分工、临时组团、资源调配、统筹兼顾, 使设备和人力得到有效利用, 人力资源实现 了弹性共享, 从而实现工作高效调度, 提高了应对效率。

3.2.3矩阵结构型高校办公室是广度和深度的“教练员”

在高校办公室工作, 既要是专才, 又要是通才。要根据各个岗位的特点和需要, 为每个 工作人员规划发展方向, 让他们成为校内工作的“权威”。矩阵结构下的办公室, 要求各科室 人员不定期、无规律的组合, 这需要人员既熟悉各自科室业务, 又要熟悉项目工作业务, 更 要学会在不同科室、部门之间的协调和沟通, 每个人员都要在全校性的活动中“指挥千军万 马”。通过一次又一次的“演练”, 每个人员可以迅速成长并最终成为独挡一面的一专多能型人 才。

\section{4.构建矩阵结构型高校办公室的工具选择}

\section{1唯物辩证法}

唯物辩证法是研究事务和思维的哲学方法, 其基本观点对高校办公室工作具有重要指导 作用和意义。使用整体论、两点论和重点论相统一的观点可以处理矩阵结构型高校办公室纵 向与横向的矛盾存在以及纷繁复杂的日常工作。办公室的工作复杂, 头绪繁多, 要突出重点、 分清主次, 同时还要着眼全局、兼顾各方, 才能收获良好的工作效果。坚持两点论和重点论 的统一, 就是要抓住办公室的重点和主流工作, 实现科室工作和项目工作的统筹兼顾。实践 证明, 毫不动摇、长期坚持并不断完善高校党委领导下的校长负责制,围绕学校的统一部署, 立德树人，服务中心、服务师生，就是抓住了办公室工作的主流和重点。

\section{2时间的定性运筹术}

矩阵结构型高校办公室其优势是可以快速高效地处置突发和临时事务, 这对时间的应用 提出了很高的要求。时间的定性运筹术要求要最大限度的谋求可控时间, 用最佳时间完成最 
重要的工作; 要具备区分重要事件和一般事件的能力, 并把常规工作标准化。高校办公室的 工作复杂多变, 工作人员易陷入忙乱的事务性工作而忽略大局。要把握全局, 不断调整工作 重心; 要合理安排好解决矛盾的先后, 对常规性、规律性的工作, 要摸索总结规律, 多些预 判和预测，尽早安排；对临时的突发性工作，要总结反思，有备无患，掌握工作主动权。

\section{3权变理论}

管理学中的权变理论认为, 对学校的管理不存在一种最好的通用方法; 组织设计和管理 方式的选择必须建立在对情景进行认真分析的基础上。高校办公室需要协调校领导、各职能 部门、各系院的关系, 受多种因素的影响, 是一个高速运转的系统。权变理论要求要以系统 的、动态的观点来理解和设计高校办公室的矩阵结构, 要努力适应不断变化的环境、战略、 人员情况，采取不同的方案和应对措施。

\section{4绩效考核}

学校管理活动中基本的群体心态是追求激励、避免挫伤。实施绩效考核是加强人才队伍 建设、促进组织发展的重要举措, 可以最大限度地调动人员干事创业的积极性, 形成各尽其 能、各得其所的良好局面。对矩阵结构型的高校办公室来说, 由于很多的工作都实行项目制, 更需要加强目标管理与量化考核, 以项目完成的多少、好坏作为衡量工作量多寡、有无的一 个重要指标。

\section{References}

[1] Song Qiang, Analysis on Administration Affairs in Colleges and Universities from the Perspective of Materialist Dialectics, Journal of Shijiazhuang Tiedao University(Social Science Edition) , vol.11, pp.106-110, 2017.

[2] $\mathrm{Hu}$ Xiaojin, and Yin Yongjian, Strengthening the six kinds of consciousness and improving the four abilities-The reflection on the office work in universities in the new circumstances, Journal of Xi'an Polytechnic University, vol.27, pp.543-546, 2013. 